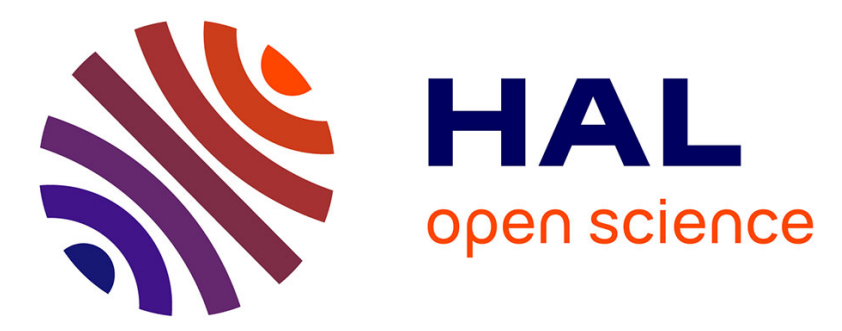

\title{
The hypothesis of the moving comb in frequency shifted feedback lasers
}

\author{
H. Guillet de Chatellus, E. Lacot, W. Glastre, O. Jacquin, O. Hugon
}

\section{To cite this version:}

H. Guillet de Chatellus, E. Lacot, W. Glastre, O. Jacquin, O. Hugon. The hypothesis of the moving comb in frequency shifted feedback lasers. Optics Communications, 2011, 284, pp.4965-4970. 10.1016/J.OPTCOM.2011.06.042 . hal-00951867

\section{HAL Id: hal-00951867 \\ https://hal.science/hal-00951867}

Submitted on 27 Feb 2014

HAL is a multi-disciplinary open access archive for the deposit and dissemination of scientific research documents, whether they are published or not. The documents may come from teaching and research institutions in France or abroad, or from public or private research centers.
L'archive ouverte pluridisciplinaire HAL, est destinée au dépôt et à la diffusion de documents scientifiques de niveau recherche, publiés ou non, émanant des établissements d'enseignement et de recherche français ou étrangers, des laboratoires publics ou privés. 


\title{
The hypothesis of the moving comb in frequency shifted feedback lasers
}

\author{
H. Guillet de Chatellus, E. Lacot, W. Glastre, O. Jacquin, O. Hugon \\ CNRS/Univ. Grenoble 1, Laboratoire Interdisciplinaire de Physique, \\ UMR 5588, Grenoble, F-38041, France
}

February 24, 2014

\begin{abstract}
The use of frequency-shifted feedback (FSF) lasers in optical metrology is based on a unique coherence property: the appearance of beats in the noise spectrum at the output of a two-beam interferometer, whose frequencies vary linearly with the path delay of the interferometer. A description of the output of a FSF laser as a moving comb of optical frequencies is generally admitted to explain these specific coherence properties. Here starting from the model of a passive FSF cavity seeded by spontaneous emission we give a rigorous description of the time-spectrum properties of FSF lasers and show that the moving comb exists only in the limit of small frequency shift.
\end{abstract}

\section{Introduction}

A frequency shifted feedback (FSF) laser is a laser cavity in which a frequency shifter -usually an acousto-optics modulator (AOM)- has been inserted [1]. FSF cavities are characterized by $\Delta$ the angular frequency of the frequency shifter and $\tau_{r}$ the cavity roundtrip time. They show some similarity with Q-switch mode locked lasers, but with the difference that the FSF cavity is closed on the first diffraction order of the AOM. Moreover the shift frequency is generally chosen independently from the cavity free spectral range. FSF lasers can be seeded by a an external laser field, or by the spontaneous emission from the gain medium of the laser cavity. Each time a photon makes a roundtrip in the cavity, it undergoes a frequency shift (usually in the $\mathrm{MHz}$ to $\mathrm{GHz}$ range), which suppresses the interference effect responsible for the formation of the optical frequency comb of multimode lasers. When the seeding arises from spontaneous emission the output optical spectrum is broadband and continuous despite the fact that the noise spectrum is discrete [2]. The interest for these lasers has been triggered by both theoretical challenges and potential applications in different fields. A detailed review of "early" demonstrations can be found in [3]. In the last ten years, the field of applications of FSF lasers have spread to various 


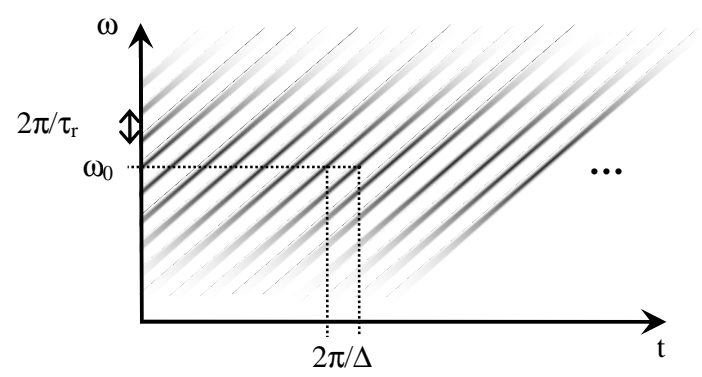

Figure 1: The moving comb model. The optical spectrum is plotted as a function of $t$.

areas, such as manipulation of atoms by laser $[4,5,6]$, optical communications $[7]$, optical frequency combs $[8,9]$ and optical frequency domain ranging $[10,11$, $12,13]$.

Most of applications of FSF lasers in optical metrology rely on a property specific to these lasers: when a FSF laser enters a two beams interferometer the noise spectrum of the laser at the output of the interferometer exhibits additional beats, whose frequencies evolve linearly with the path delay of the interferometer. This property remains valid for path delays orders of magnitude larger than the coherence length of the laser [14]. In 1993 experimental studies carried out on a FSF laser in the limit of a small frequency shift (i.e. $\Delta \ll 2 \pi / \tau_{r}$ ) have demonstrated that the output field of a FSF laser is a chirping comb of frequencies or moving comb (MC), consisting in the superposition of optical modes whose instantaneous frequency chirps with time (Fig. 1) [15]. As the instantaneous frequency changes with time the amplitude of the mode evolves according to the optical spectral density of the FSF laser. The MC shows a periodicity of $2 \pi / \Delta$ and $1 / \tau_{r}$ in the time and frequency domain respectively. The $\mathrm{MC}$ model gives an intuitive understanding of the RF properties of FSF lasers: at the output of the two-beam interferometer the optical spectrum consists in a system of two moving combs shifted by the time delay of the interferometer and the beat frequencies in the noise spectrum are directly deduced from the differences between the instantaneous optical frequencies of the modes of the two combs [12]. Numerical simulations tend to prove the existence of such a comb even when the small shift approximation does not hold [16].

Therefore the observation of additional beats at the output of the Michelson interferometer has become systematically interpreted as the signature of a chirping frequency comb [17]. In the case of a monochromatic seeding the pertinence of the MC model has been demonstrated [18]. However the MC model lacks a rigorous justification in the case of a seeding by spontaneous emission. Moreover it can be pointed out that the description of the FSF laser field by the moving comb is abusive in most experimental cases. From Fourier analysis the 
size of an elementary cell in the time-frequency representation is equal to unity [19]. To resolve an element of the comb, the integration time must be much smaller than $2 \pi / \Delta$ and the corresponding frequency bandwidth is then larger than $\Delta / 2 \pi$. However to resolve the comb the frequency bandwidth should be much smaller than $1 / \tau_{r}$. Therefore the time-frequency $\mathrm{MC}$ has a physical reality only in the case where $\Delta / 2 \pi \ll 1 / \tau_{r}$ that is in the small shift approximation: it appears that the MC model remains a convenient - but improper - hypothesis.

Surprisingly a deeper theoretical analysis on FSF lasers seeded with stochastic noise has recently led to an unexpected demonstration: there is no need to consider the MC model to account for the additional RF beats observed at the output of a Michelson interferometer [18, 20]. Authors consider a passive cavity $(\mathrm{PC})$ seeded by a stochastic field representing the spontaneous emission in the spatial mode of the laser, and provide an analytical expression of the noise spectrum of a FSF laser, including the additional beats at the output of the interferometer, which seems to rule out the generally admitted hypothesis of the MC model.

In this paper we focus on the apparent contradiction between the $\mathrm{MC}$ model [15] and the PC model seeded by a stochastic field [20]. Starting from the PC model in the time-frequency representation we show that there is indeed a moving comb but only in the limit of small frequency shift. More generally we show that the spectral density of the FSF light field in the time-frequency representation involves a universal class of periodic functions defined by their Fourier series. This class of functions only depend from the parameters of the cavity. Their specificity lies in the fact that the phase of the $n^{t h}$ spectral Fourier component evolves quadratically with $n$ : in the limit of a small frequency shift, this class of functions tends to a discrete comb which justifies the MC model in that case. It exhibits a more complicated behavior in the general case, where it shows a close connection to Gauss sums [21]. We also evidence a correspondence with the Talbot effect in optical diffraction [22]. Finally we link the fact that FSF lasers show both a continuous optical spectrum and a discrete noise (or $\mathrm{RF}$ ) spectrum, to the intrinsic properties of this class of functions.

\section{The passive cavity model}

\section{$2.1 \quad$ Presentation}

The passive cavity model (PC) involves a ring-like optical cavity closed on the first order of diffraction of an acousto-optics (AO) modulator. For more consistence we adopt the notations used in [20] and [23]. The AO modulator is driven at angular frequency $\Delta$ : each time a photon makes a roundtrip in the cavity it experiences a frequency shift equal to $\Delta / 2 \pi . \tau_{r}$ is the roundtrip time in the cavity and we define $R$ as the reflection coefficient for the whole cavity. $R$ is slightly smaller than one to account for diffraction losses in the AO modulator and partial reflectivity of the output mirror. We neglect any frequency-dependent gain or losses and consider a flat transmission function for any frequency component 


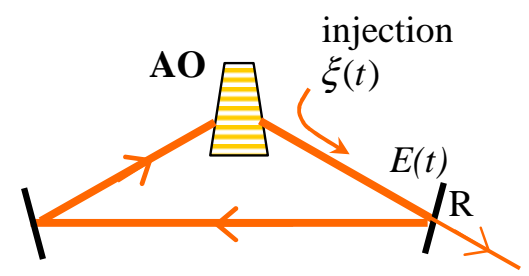

Figure 2: Sketch of the passive cavity model (PCM).

of the intracavity light field.

This cavity is injected by an optical field $\xi$ which represents the generic seeding of the passive cavity (spontaneous emission, monochromatic or phasemodulated wave...). We consider $E(t)$ as the light field just before the output coupler of the passive cavity. The amplitude of the seeding at the same position is named $\xi(t)$. The laser field obeys the simple recurrence relationship:

$$
E(t)=\xi(t)+R e^{i \Delta t} E\left(t-\tau_{r}\right)
$$

which leads to [20]:

$$
E(t)=\sum_{n=0}^{\infty} R^{n} \xi\left(t-n \tau_{r}\right) e^{i\left(n \Delta t-\Phi_{n}\right)}
$$

where $\Phi_{n}=\Delta \tau_{r} \sum_{l=0}^{n-1} l=\frac{n(n-1)}{2} \Delta \tau_{r}$.

One can define $n_{0}$ as the smallest integer verifying $R^{n_{0}} \leq 0.01$ i.e. $n_{0} \approx$ $4.6 / \ln (1 / R)$. Therefore when necessary one can approximate the FSF light field by the truncated sum:

$$
E(t)=\sum_{n=0}^{n_{0}} R^{n} \xi\left(t-n \tau_{r}\right) e^{i\left(n \Delta t-\Phi_{n}\right)} .
$$

\subsection{Optical spectrum of FSF lasers}

Without loss of generality we define the seeding field by its Fourier transform:

$$
\xi(t)=\int \tilde{\xi}(\omega) e^{i \omega t} d \omega
$$

where:

$$
\tilde{\xi}(\omega)=\frac{1}{2 \pi} \int \xi(t) e^{-i \omega t} d t .
$$

The spectrum of the FSF laser field writes: 


$$
\tilde{E}(\omega)=\frac{1}{2 \pi} \sum_{n=0}^{\infty} R^{n} e^{-i \Phi_{n}} \int \xi\left(t-n \tau_{r}\right) e^{-i(\omega-n \Delta) t} d t
$$

and then:

$$
\tilde{E}(\omega)=\sum_{n=0}^{\infty} R^{n} \tilde{\xi}(\omega-n \Delta) e^{-i n \omega \tau_{r}} e^{i \Phi_{n+1}} .
$$

For a seeding by spontaneous emission, the spectrum $\tilde{\xi}(\omega)$ is a delta-correlated process. For a given $\omega, \tilde{E}(\omega)$ is a sum of incoherent terms with random phases. Therefore $\tilde{E}(\omega)$ is a delta-correlated Rayleigh density function [24]. The optical spectrum of FSF lasers appears therefore as broadband and continuous, or modeless [2]. In the case where the variations of $|\tilde{\xi}(\omega)|$ with $\omega$ occur on a scale much larger than $n_{0} \Delta$, we have:

$$
\tilde{E}(\omega) \approx|\tilde{\xi}(\omega)| \sum_{n=0}^{\infty} R^{n} e^{-i \psi_{n}(\omega)}
$$

where $\psi_{n}(\omega)$ is a random distribution of phases between $-\pi$ and $+\pi$. The summation term is therefore the sum of phasors with negative exponential amplitude and random phase. The second momentum of the negative exponential amplitude distribution is independent from $\omega$ and is equal to $1 /(2 \ln (1 / R))$. $\tilde{E}(\omega)$ obeys a Rayleigh probability density function and the ensemble average of the optical power spectrum of the FSF laser simply writes [24]:

$$
<|\tilde{E}(\omega)|^{2}>\approx \frac{|\tilde{\xi}(\omega)|^{2}}{2 \ln (1 / R)} .
$$

Then the spectrum of the FSF laser reproduces the spectrum of the seeding field.

\section{Time-frequency representation of FSF lasers}

We now consider the time-dependent spectral properties of FSF lasers. We write the FSF laser field as:

$$
\begin{aligned}
E(t) & =\sum_{n=0}^{\infty} R^{n} e^{-i \Phi_{n}} \int \tilde{\xi}(\omega) e^{i(\omega+n \Delta) t} e^{-i n \omega \tau_{r}} d \omega \\
& =\int \tilde{\xi}(\omega) F_{R, \Delta \tau_{r}}\left(\omega \tau_{r}-\Delta t\right) e^{i \omega t} d \omega
\end{aligned}
$$

where $F_{R, \phi}$ is a $2 \pi$ periodic function defined by its Fourier series:

$$
F_{R, \phi}(\theta)=\sum_{n=0}^{\infty} R^{n} e^{-i n \theta} e^{-i n(n-1) \phi / 2}
$$


The amplitude of the Fourier coefficients decreases exponentially while their phase evolves quadratically with $n$.

\subsection{Interpretation}

A remarkable consequence arising from the previous expression is the decoupling between the intrinsic properties of the seeding field given by its Fourier transform $\tilde{\xi}(\omega)$, from the specific properties of the passive cavity which are represented by the universal class of functions $F_{R, \phi}$. The latter only depends from the physical parameters of the cavity $R, \Delta$ and $\tau_{r}$. At first sight the term $\tilde{\xi}(\omega) F_{R, \Delta \tau_{r}}\left(\omega \tau_{r}-\right.$ $\Delta t$ ) can be interpreted as the time-dependent Fourier transform of the FSF laser field. In a time-frequency representation, the function $F_{R, \Delta \tau_{r}}\left(\omega \tau_{r}-\Delta t\right)$ is periodic with both $t$ and $\omega$, with the periods $2 \pi / \Delta$ and $2 \pi / \tau_{r}$ respectively in the time and angular frequency domains. It can be seen that this function is shifting with time in the time-frequency plane at a rate equal to $\frac{\delta \omega}{\delta t}=\frac{\Delta}{\tau}$ which illustrates intuitively the intrinsic chirped nature of the FSF laser field. It is worth noting that due to the quadratic phase of its Fourier components the $F_{R, \phi}$ function differs in the general case from a comb of lines, which invalidates intuitively the picture of the moving comb (fig. 3).

An interesting parallel can be made with the diffraction phenomenon: this expression is the restriction to negative frequencies of the field diffracted by a grating of equidistant narrow lines, leading to the Talbot effect, i.e. the repetition of the diffracting pattern at periodic distances from the grating [22]. Fig. 4 gives a plot of $\left|F_{R, \phi}(\theta)\right|^{2}$ as a function of $\theta$ and $\phi$ and shows a close analogy with the so-called Talbot carpet. Interestingly the expression of $F_{2 \pi}$ is also close to the Gauss sum used for factorizing numbers, which could lead to further applications of FSF lasers [21].

Finally the functions $F_{R, \phi}(\theta)$ have a physical reality when instead of a spontaneous emission, the FSF cavity is seeded by a monochromatic laser field defined by its spectrum $\tilde{\xi}(\omega)=E_{0} \delta\left(\omega-\omega_{0}\right)$. The FSF laser field simply writes $E(t)=E_{0} F_{R, \Delta \tau_{r}}\left(\omega_{0} \tau_{r}-\Delta t\right) e^{i \omega_{0} t}$ and the intensity: $I(t)=\left|E_{0}\right|^{2} \mid F_{R, \Delta \tau_{r}}\left(\omega_{0} \tau_{r}-\right.$ $\Delta t)\left.\right|^{2}$. $I(t)$ directly reproduces the square modulus of $F_{R, \phi}(\theta)$. We therefore simply recover from the analogy with the Talbot effect, why FSF lasers seeded by a monochromatic field emit in pulsed modes when $\Delta / 2 \pi$ is commensurable with $1 / \tau_{r}[1,18]$.

\subsection{Instantaneous spectrum of FSF lasers}

A rigorous calculation of the instantaneous spectrum of the passive FSF cavity requires a short time Fourier transform (STFT). We consider for instance $w(t)$ a real Gaussian window function centered at $t=0$, defined by $T_{w}$, the full-width at half maximum (FWHM) and the normalization condition:

$$
\int w(t) d t=1 .
$$

We define $\tilde{w}$ as its Fourier transform: 

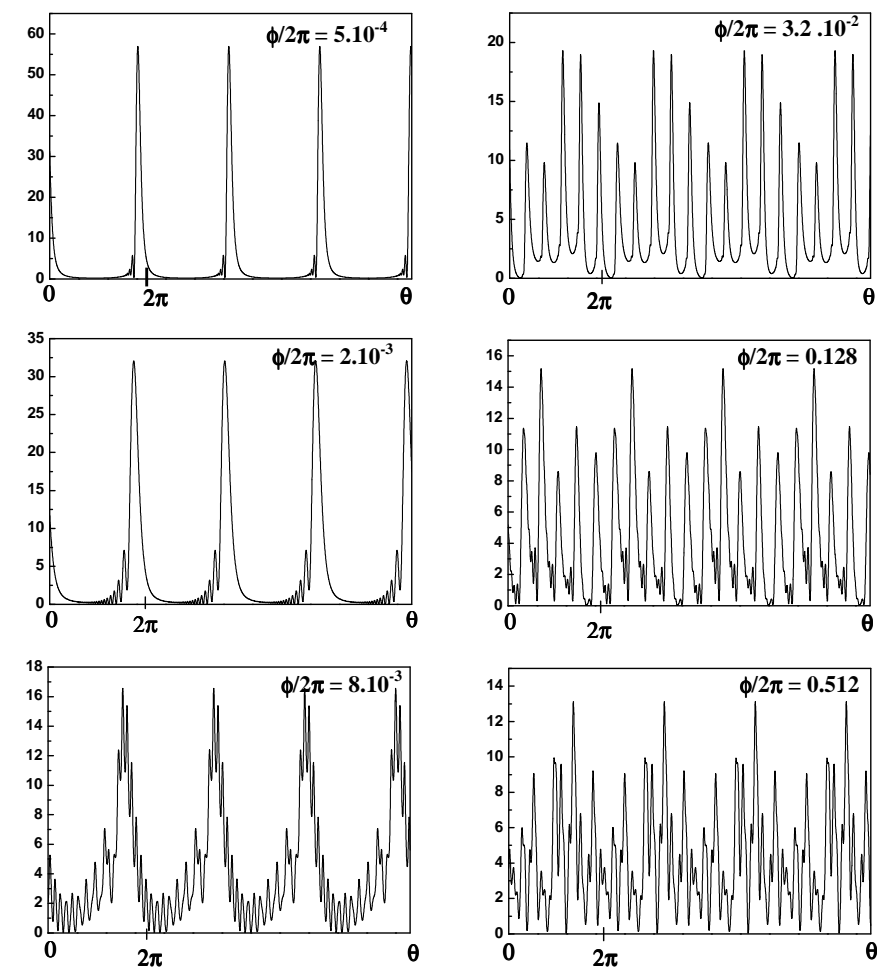

Figure 3: Plot of $\left|F_{R, \phi}(\theta)\right|^{2}$ for $R=0.9 . \quad \phi$ increases from $2 \pi \times 510^{-4}$ to $2 \pi \times 0.512$. 


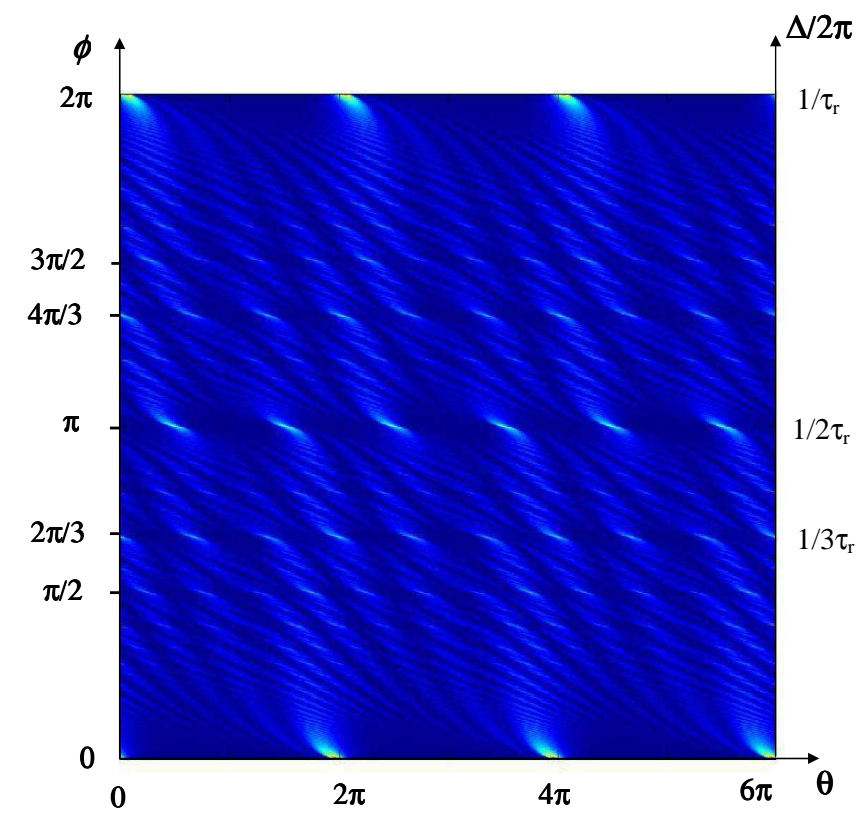

Figure 4: Talbot carpet-like surface plot of $\left|F_{R, \phi}(\theta)\right|^{2}$ for $R=0.9$. When $\phi / \pi$ is a rational $\left|F_{R, \phi}(\theta)\right|^{2}$ is a comb-like function of $\theta$. In the case $\phi \simeq 0$ (i.e. in the limit of a small frequency shift), $\left|F_{R, \phi}(\theta)\right|^{2}$ tends to a discrete comb. 


$$
\tilde{w}(\omega)=\frac{1}{2 \pi} \int w(t) e^{-i \omega t} d t
$$

In the angular frequency space the FWHM of $\tilde{w}$ is equal to $\frac{\sqrt{8 \ln 2}}{T_{\omega}} \simeq \frac{2.35}{T_{\omega}}$. The STFT at time $\tau$ of the FSF light field is:

$$
\begin{aligned}
& \tilde{E}(\tau, \omega)=\int d t w(t-\tau) E(t) e^{i \omega t} \\
& =\sum_{n=0}^{\infty} R^{n} e^{-i \Phi_{n}} \\
& \times \int d t \int d \omega^{\prime} w(t-\tau) \tilde{\xi}\left(\omega^{\prime}\right) e^{i \omega^{\prime}\left(t-n \tau_{r}\right)+i(\omega+n \Delta) t} .
\end{aligned}
$$

Taking $\Omega=\omega+\omega^{\prime}$ leads to:

$$
\begin{aligned}
& \tilde{E}(\tau, \omega)=\sum_{n=0}^{\infty} R^{n} e^{-i \Phi_{n}} \\
& \times \int d \Omega \tilde{\xi}(\Omega-\omega) e^{i(\omega-\Omega) n \tau_{r}} \int d t w(t-\tau) e^{i(\Omega+n \Delta) t} .
\end{aligned}
$$

We choose the width $T_{w}$ of the window function so that $n_{0} \Delta T_{w} \ll 2 \pi$, for instance $T_{w}=2 \pi /\left(100 n_{0} \Delta\right)$. In that case $\tilde{E}(\tau, \omega)$ is equal to:

$$
\begin{aligned}
& \tilde{E}(\tau, \omega)=\sum_{n=0}^{\infty} R^{n} e^{-i \Phi_{n}} \\
& \times \int d \Omega \tilde{\xi}(\Omega-\omega) e^{i(\omega-\Omega) n \tau_{r}+i n \Delta \tau} \int d t w(t-\tau) e^{i \Omega t}
\end{aligned}
$$

which rewrites as:

$$
\begin{aligned}
& \tilde{E}(\tau, \omega)=\int d \Omega \tilde{\xi}(\Omega-\omega) F_{R, \Delta \tau_{r}}\left((\Omega-\omega) \tau_{r}-\Delta \tau\right) \\
& \times \int d t w(t-\tau) e^{i \Omega t}
\end{aligned}
$$

and finally:

$$
\begin{aligned}
& \tilde{E}(\tau, \omega)= \\
& \int d \Omega \tilde{\xi}(\Omega-\omega) F_{R, \Delta \tau_{r}}\left((\Omega-\omega) \tau_{r}-\Delta \tau\right) \tilde{w}(\Omega) e^{i \Omega \tau} .
\end{aligned}
$$

We obtain the convolution product of $\tilde{\xi}(\omega) F_{R, \Delta \tau_{r}}\left(\omega \tau_{r}-\Delta \tau\right)$ by the function $\tilde{w}(\omega) e^{i \omega \tau}$ : the instantaneous spectrum at time $\tau$ is equal to the product $\tilde{\xi}(\omega) F_{R, \Delta \tau_{r}}\left(\omega \tau_{r}-\Delta \tau\right)$ convoluted in the Fourier space by a Gaussian function of width $\frac{2.35}{T_{\omega}}=37 n_{0} \Delta$. 


\subsection{Limit of small frequency shift}

We now turn to the situation where the frequency shift is much smaller than the cavity free spectral range. This case was investigated experimentally and led to the model of the moving comb model [15].

We suppose that $\Delta$ is small enough so that the cumulated phase over $n_{0}$ roundtrips $\frac{\Delta \tau_{r}}{2} n_{0}\left(n_{0}-1\right)$ is much smaller than $2 \pi$. We consider for instance $\frac{\Delta \tau_{r}}{2} n_{0}\left(n_{0}-1\right)=2 \pi / 100$. In that case:

$$
E(t) \approx \int \tilde{\xi}(\omega) \frac{e^{i \omega t}}{1-R e^{-i\left(\omega \tau_{r}-\Delta t\right)}} d \omega .
$$

The Airy function can be described as a comb of equidistant Lorentzian lines $[25]$ :

$$
\begin{aligned}
& \Lambda_{R}(\theta)=\frac{1}{1-R e^{-i \theta}}=\frac{1}{(1-R)^{2}} \\
& \times \sum_{k=-\infty}^{k=+\infty} \frac{1}{1+\frac{2 R}{(1-R)^{2}}(\theta-k \pi)^{2}}[1-R \cos \theta-i R \sin \theta] .
\end{aligned}
$$

The FWHM of the individual Lorentzian lines is equal to $\sqrt{2} \frac{1-R}{\sqrt{R}}$. The instantaneous spectrum of the FSF light field is therefore the convolution product of $\tilde{\xi}(\omega) \Lambda_{R}\left(\omega \tau_{r}-\Delta \tau\right)$ by a gaussian function of width $37 n_{0} \Delta$. Taking into account $\frac{\Delta \tau_{r}}{2} n_{0}\left(n_{0}-1\right)=2 \pi / 100$ and the definition of $n_{0}$, it is easy to show

that $37 n_{0} \Delta \approx \frac{1-R}{\tau_{r}}$, that is to say comparable or smaller than the width of the individual Lorentzian components in the angular frequency representation. Therefore the instantaneous spectrum of the FSF laser in the limit of a small frequency shift consists effectively in a moving comb with the periods $2 \pi / \Delta$ and $2 \pi / \tau_{r}$, in the time and angular frequency domains respectively. Time-frequency representations of the FSF field are plotted on fig. 5 for different values of the product $\Delta \tau_{r} / 2 \pi$. Note that the contrast of the moving comb vanishes when $\Delta \tau_{r} / 2 \pi>10^{-2}$ and the instantaneous spectrum appears then as a continuum.

\section{Noise properties of FSF lasers}

We now turn to the calculation of the noise (or RF) properties of FSF lasers seeded by spontaneous emission. It has been observed than although the optical spectrum is broadband and continuous the noise spectrum is discrete [20]. This property is specific to the class of functions $F_{R, \phi}$ and actually relies on the quadratic phase of its Fourier components.

We calculate the product $E(t) E^{*}(t+\tau)$ :

$$
E(t) E^{*}(t+\tau)
$$



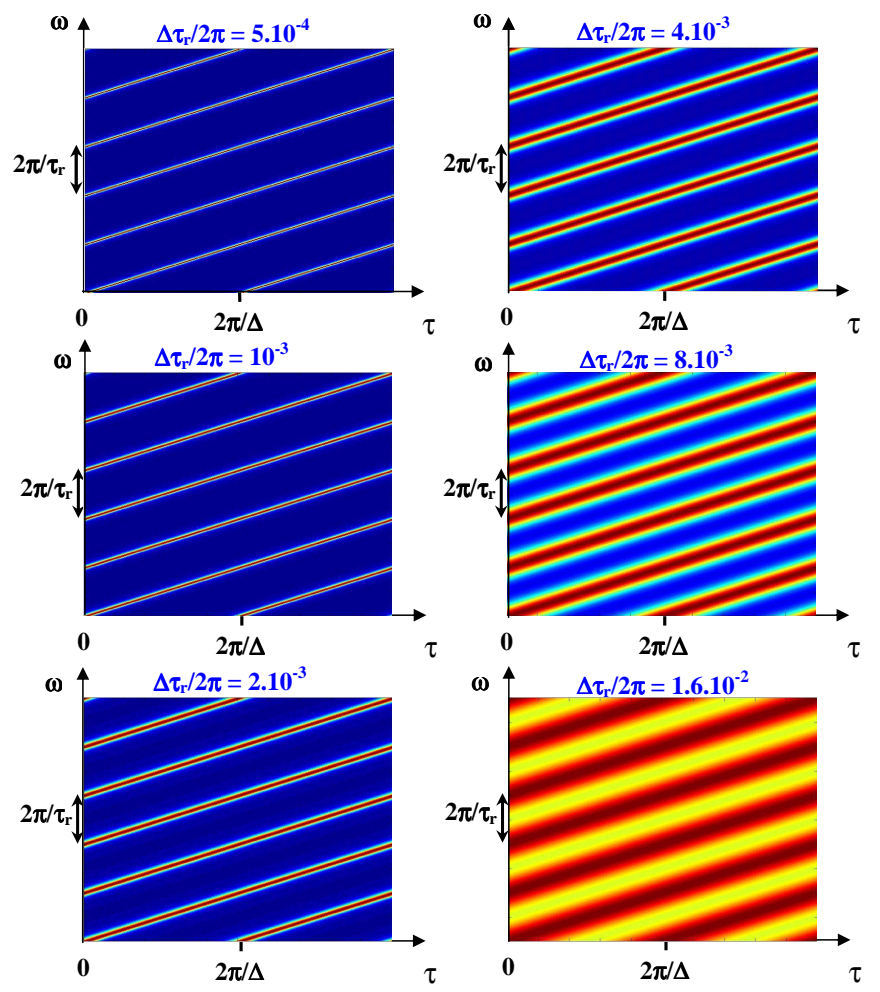

Figure 5: Time frequency representation of the FSF output field for different values of the frequency shift $\Delta$ compared to the cavity free spectral range $1 / \tau_{r}$. We consider a flat spectrum for the seeding $(\tilde{\xi}(\omega)=1)$ and a reflection coefficient $R=0.9$. The color scale is adjusted between 0 (dark blue) and the maximum of the surface plot (red). 


$$
\begin{aligned}
& =\iint d \omega d \omega^{\prime} \tilde{\xi}(\omega) \tilde{\xi}^{*}\left(\omega^{\prime}\right) e^{i\left(\omega-\omega^{\prime}\right) t} e^{-i \omega^{\prime} \tau} \\
& \times F_{R, \Delta \tau_{r}}\left(\omega \tau_{r}-\Delta t\right) F_{R, \Delta \tau_{r}}^{*}\left(\omega^{\prime} \tau_{r}-\Delta t-\Delta \tau\right) .
\end{aligned}
$$

We have:

$$
\begin{aligned}
& F_{R, \Delta \tau_{r}}\left(\omega \tau_{r}-\Delta t\right) F_{R, \Delta \tau_{r}}^{*}\left(\omega^{\prime} \tau_{r}-\Delta t-\Delta \tau\right) \\
& =\sum_{n=0}^{\infty} \sum_{p=0}^{\infty} R^{n+p} e^{-i\left(n \omega-p \omega^{\prime}\right) \tau_{r}} e^{i(n-p) \Delta t} e^{-i p \Delta \tau} e^{-i\left(\Phi_{n}-\Phi_{p}\right)} .
\end{aligned}
$$

Recall that:

$$
\sum_{n=0}^{\infty} \sum_{p=0}^{\infty} a_{n, p}=\sum_{p=0}^{\infty} \sum_{q=0}^{\infty} a_{p+q, p}+\sum_{n=0}^{\infty} \sum_{q=0}^{\infty} a_{n, n+q}-\sum_{q=0}^{\infty} a_{q, q}
$$

which gives three contributions. Taking $n=p+q$ the first contribution to the product $E(t) E^{*}(t+\tau)$ writes:

$$
\begin{aligned}
& \iint d \omega d \omega^{\prime} \tilde{\xi}(\omega) \tilde{\xi}^{*}\left(\omega^{\prime}\right) e^{i\left(\omega-\omega^{\prime}\right) t} e^{-i \omega^{\prime} \tau} \\
& \times \sum_{q=0}^{\infty} R^{q} e^{-i q\left(\omega \tau_{r}-\Delta t\right)} e^{-i \frac{q(q-1)}{2} \Delta \tau_{r}} \sum_{p=0}^{\infty} R^{2 p} e^{-i p\left[\left(\omega-\omega^{\prime}+q \Delta\right) \tau_{r}+\Delta \tau\right]} .
\end{aligned}
$$

Taking $\Omega=\omega-\omega^{\prime}+q \Delta$ this expression is rewritten as:

$$
\begin{aligned}
& \int\left[\sum_{q=0}^{\infty} R^{q} e^{-i \Phi_{q}} e^{-i q \Delta \tau}\right. \\
& \left.\times \int d \omega \tilde{\xi}(\omega) \tilde{\xi}^{*}(\omega+q \Delta-\Omega) e^{-i \omega\left(q \tau_{r}+\tau\right)}\right] \\
& \times e^{i \Omega(t+\tau)} \Lambda_{R^{2}}\left(\Omega \tau_{r}+\Delta \tau\right) d \Omega .
\end{aligned}
$$

The appearance of the comb-like function $\Lambda_{R^{2}}$ in this expression is a consequence of the quadratic phase of the Fourier components of $F_{R, \theta}$. It can be checked that this property is also specific to the quadratic phase [26]. When the three contributions are added one obtains the expression of the product $E(t) E^{*}(t+\tau)$ :

$$
E(t) E^{*}(t+\tau)=\int G(\Omega) e^{i \Omega(t+\tau)} \Lambda_{R^{2}}\left(\Omega \tau_{r}+\Delta \tau\right) d \Omega
$$

where: 


$$
\begin{aligned}
& G(\Omega)=\sum_{q=-\infty}^{\infty} R^{|q|} \eta^{q} \\
& \times \int \tilde{\xi}(\omega) \tilde{\xi}^{*}(\omega+q \Delta-\Omega) e^{-i \omega\left(q \tau_{r}+\tau\right)} d \omega
\end{aligned}
$$

and where $\eta=e^{-i \Delta \tau}$ when $q \geq 0$ and $\eta=e^{-i \Omega \tau_{r}}$ when $q<0$.

The product $E(t) E^{*}(t+\tau)$ is therefore defined by its Fourier transform. When $\tau=0$, this quantity reduces to the intensity of the FSF laser. Then the noise spectrum of the laser consists in a comb of equidistant Lorentzian lines separated by the cavity free spectral range $1 / \tau_{r}[20]$, multiplied by the $G(\Omega)$ term. We retrieve here from the passive cavity model, why the noise spectrum of a FSF laser seeded by spontaneous emission shows equidistant peaks separated by the cavity free spectral range, despite the fact that the optical spectrum is broadband and continuous. When $\tau \neq 0$, the product $E(t) E^{*}(t+\tau)$ appears in the expression of the intensity at the output of the interferometer with the time delay $\tau$. We recover here the additional beats in the noise spectrum at frequencies shifted by $\Delta \tau / \tau_{r}$ from the multiples of the free spectral range of the FSF laser cavity [20].

\section{Conclusion}

In this letter we have thrown a new light on the unique properties of frequency shifted feedback lasers and discussed the question of the existence of the moving comb in these lasers and its compatibility with the passive cavity model. Starting from a time-frequency representation of the FSF laser field in the passive cavity model, we have shown that the latter can be written in a form that decouples the intrinsic parameters of the cavity from the properties of the seeding field. In this paper we focused more particularly on the passive cavity seeded by spontaneous emission from the gain medium but this approach can be extended to single mode or phase-modulated seeding. We have shown that the FSF laser field involves a class of functions $F_{R, \phi}$ characterized by the parameters of the cavity and defined by its Fourier series. The phase of the $n^{\text {th }}$ Fourier component evolves quadratically with $n$. We have shown that the instantaneous spectrum of the FSF laser consists in a moving comb only in the limit of the small frequency shift. As soon as the frequency shift exceeds a few percents of the cavity free spectral range, the frequency comb is washed out and the instantaneous spectrum appears continuous for two reasons: the uncertainty principle in the time-frequency plane, and the fact that $F_{R, \Delta \tau_{r}}$ differs from a comb when $\Delta \tau_{r}>0$ (with notable exceptions when $\Delta \tau_{r}$ is commensurable with $\pi$, where $F_{R, \Delta \tau_{r}}$ recovers then a discrete shape). Therefore the practical hypothesis of the moving comb in FSF lasers is most of the time abusive. Finally we showed that the quadratic phase of the Fourier components of $F_{R, \phi}$ is responsible for the fact that the noise spectrum of FSF lasers is discrete contrary to its broadband and continuous instantaneous optical spectrum. 


\section{References}

[1] F. V. Kowalski, J. A. Squier, J. T. Pinckney, Appl. Phys. Lett. 50 (1987) 711.

[2] F. V. Kowalski, P. D. Hale, S. J. Shattil, Opt. Lett. 13 (1988) 622.

[3] J. M. Sousa, O. G. Okhotnikov, Opt. Comm. 183 (2000) 227.

[4] J.-P. Pique, S. Farinotti, J. Opt. Soc. Am. B 20 (2003) 2093.

[5] H. Guillet de Chatellus, J.-P. Pique, Opt. Lett. 34 (2009) 755.

[6] V. I. Romanenko, A. V. Romanenko, L. P. Yatsenko, G. A. Kazakov, A. N. Litvinov, B. G. Matisov, Y. V. Rozhdestvensky, J. Phys. B . 43 (2010) 215402 .

[7] F. V. Kowalski, C. Ndiaye K. Nakamura, H. Ito, Opt. Comm. 231 (2004) 149.

[8] H. Y. Ryu, H. S. Moon, H. S. Suh, Opt. Exp. 15 (2007) 11396.

[9] M. Nikodem, K. Abramski. Opt. Comm. 283 (2010) 2202.

[10] K. Nakamura, T. Hara, M. Yoshida, T. Miyahara, H. Ito, IEEE J. Quant. Elec. 36 (2000) 305.

[11] V. V. Ogurtsov, L. P. Yatsenko, V. M. Khodakovskyy, B. W. Shore, G. Bonnet, K. Bergmann, Opt. Comm. 266 (2006) 266.

[12] S. Reza, R. Ricken, V. Quiring, W. Sohler, EPS-QEOD Europhoton Conference, Pisa/Italy (2006).

[13] M. F. Brandl, O. D. Muecke, Opt. Lett. 32 (2010) 4223.

[14] K. Nakamura, T. Miyahara, H. Ito, Appl. Phys. Lett. 72 (1998) 2631.

[15] S. Balle, I. C. M. Littler, K. Bergmann, F. V. Kowalsi, Opt. Comm. 102 (1993) 166.

[16] K. Kasahara, K. Nakamura, H. Ito, IEEE J. Quant. Elec. 34 (1998) 190.

[17] A. Yoshizawa, H. Tsuchida, Opt. Comm. 155 (1998) 51.

[18] L. P. Yatsenko, B. W. Shore, K. Bergmann, Opt. Comm. 236 (2004) 183.

[19] F. V. Kowalski, S. Balle, I. C. M. Littler, K. Bergmann, Opt. Eng. 33 (1994) 1146.

[20] L. P. Yatsenko, B. W. Shore, K. Bergmann, Opt. Comm. 282 (2009) 300.

[21] D. Bigourd, B. Chatel, W. P. Schleich, B. Girard, Phys. Rev. Lett. 100 (2008) 030202. 
[22] P. Latimer, R. F. Crouse, Appl. Opt. 31 (1992) 80.

[23] H. Guillet de Chatellus, J.-P. Pique, Opt. Comm. 283 (2010) 1971.

[24] J. W. Goodman, Statistical Optics (Wiley Classics Library 2000).

[25] J. B. Kumer, W. G. Uplinger, Appl. Opt. 22 (1983) 3675.

[26] Actually if the phase of the Fourier components of $F_{R, \phi}(\theta)$ were linear with $n$, that is in the form $\Phi_{n}=n \phi$, the light field would show the same expression as in the limit of a small frequency shift, i.e. it would consist in a moving comb. Then both the (instantaneous) optical and the noise spectrum would be discrete. 